\title{
Direct reprogramming of porcine fibroblasts to neural progenitor cells
}

Dear Editor,

Neural progenitor cells (NPCs) have proven potential to facilitate mechanistic studies of neurological disorders in vitro, as well as the discovery of new medicines. In addition, NPCs have been proposed as promising cell sources for cell replacement therapy of neurological diseases (Liu et al., 2012b). For these areas of study, experimental animals are indispensable models. Among the possible animal species, pigs are advantageous compared to rodents because of their physiological and anatomical similarities to humans (Lind et al., 2007). Despite the shown advantages of porcine models in different fields, their applications are significantly restricted due to the limited access of porcine cells, including NPCs. To date, encouraging breakthroughs have been made in obtaining NPCs from a series of species by different methods, including primary cell isolation from tissues, differentiation from pluripotent stem cells, and direct reprogramming from other somatic cells (Vierbuchen et al., 2010; Giorgetti et al., 2012; Lujan et al., 2012; Thier et al., 2012; Zhang et al., 2013).

Here, we report the successful generation of induced porcine NPCs (ipNPCs) from porcine fetal fibroblasts (PFFs) (Fig. S1A, upper panel). Using our method, functional ipNPCs can be readily obtained via direct cell reprogramming without going through a pluripotent state. We show that ipNPCs retain the ability for long-term culture and efficient neural differentiation in vitro. Moreover, ipNPCs could effectively integrate into the local neural network after cell transplantation in vivo.

In order to initiate the direct cell reprogramming, we sought to prime PFFs using non-integrative episomal vectors expressing reprogramming factors (Oct4, Sox2, KIf4, Lin28, and L-Myc) (Li et al., 2011) and then subjected the cells to human embryonic stem cell-amenable culture conditions (Liu et al., 2011). Three weeks later, $\sim 10$ colonies emerged from $5 \times 10^{5}$ transduced PFFs plated on mouse embryonic fibroblasts (MEFs). These colonies did not exhibit characteristic morphology of porcine induced pluripotent stem cells (iPSCs) including high nucleus to cytoplasm ratio and clear colony boundaries, as reported in porcine iPSCs generated by viral vector-mediated methods (Ezashi et al., 2009; Wu et al., 2009). Instead, they exhibited irregular and unclear boundaries with filament-like cells spreading out (Fig. S1A, bottom panel). When a treatment of $50 \mu \mathrm{mol} / \mathrm{L}$ sodium butyrate (an inhibitor of histone deacetylases) was applied during the reprogramming, a slightly higher number of colonies with similar morphology emerged (data not shown). Immunofluorescence staining showed that these colonies expressed Sox2 at low levels and were negative for pluripotency markers Oct4 and Nanog (Fig. S1B). To initiate the neural commitment, these colonies were mechanically picked and seeded to MEFs supplied with neural stem cell culture medium (NSM) (Liu et al., 2012a). After a 10-day induction in NSM, the formation of neural rosettes was observed (Fig. 1A), which resembles an early stage of neurodevelopment. Immunofluorescence staining of neural rosettes confirmed the presence of an ipNPC population by expression of both Sox2 and Nestin (Fig. 1A). These neural rosette ipNPCs were then individualized, and subcultured in NSM on Matrigel. Under this condition, ipNPCs expanded in monolayer and over $95 \%$ of them were positive for Pax6 (Fig. 1B). Quantitative real-time PCR (qPCR) analysis further demonstrated the induction of NPC markers (NCAM, Nestin, and Pax6) in ipNPCs compared to their parental PFFs (Fig. 1C). We further determined the possible presence of residual or integrated episomal vectors in ipNPCs by genomic qPCR analysis of EBNA-1, a viral element of episomal vectors originating from Epstein-Barr virus (Li et al., 2011). The results showed that ipNPCs and PFFs contained almost undetectable levels of EBNA-1 ( 0.0001-0.001 copies per cell), while episomal vector-transfected PFFs showed nearly 100 copies per cell, implying the absence of EBNA-1 in the ipNPC genome (Fig. S1C). The negative readout from this analysis relieved safety concerns to use ipNPCs for cell transplantation in the future. Also, ipNPCs had been robustly maintained for over 12 passages without significant signs of losing potency, suggesting an ability of long term selfrenewal of ipNPCs in vitro. Meanwhile, when cultured on low-attachment plates, ipNPCs formed neurospheres spontaneously (Fig. S1D), further demonstrating neural stem cell identity and robust viability.

Next, in order to assess the neural differentiation potency of ipNPCs, in vitro spontaneous neural differentiation was performed. After three weeks of culturing in spontaneous neural differentiation medium (NDM), the cell bodies of most cells were clustered and long neurites protruded. 
A
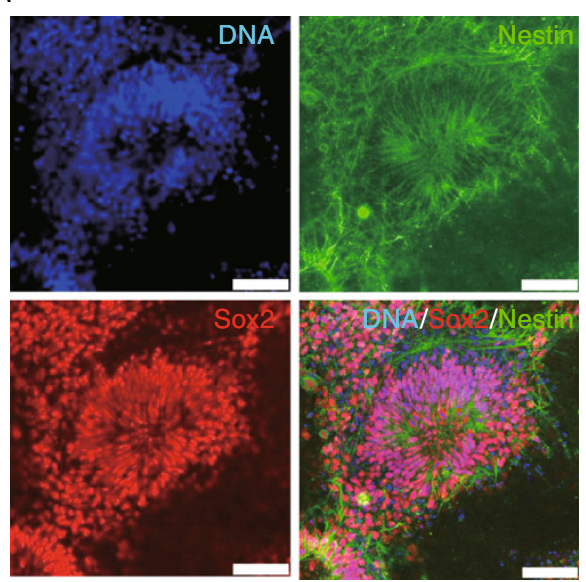

B
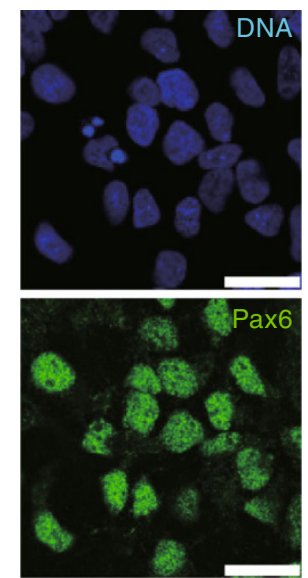

D
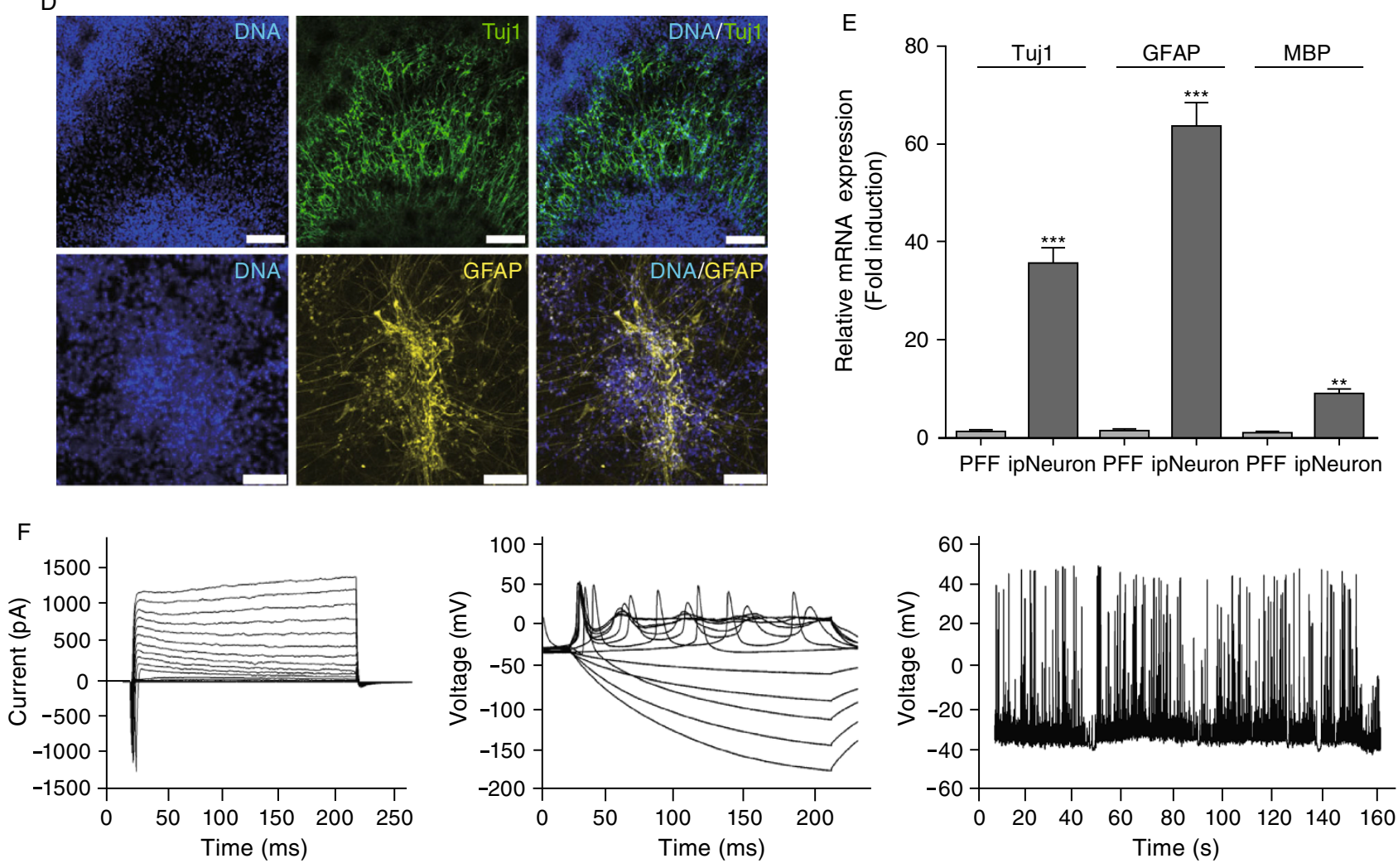

G
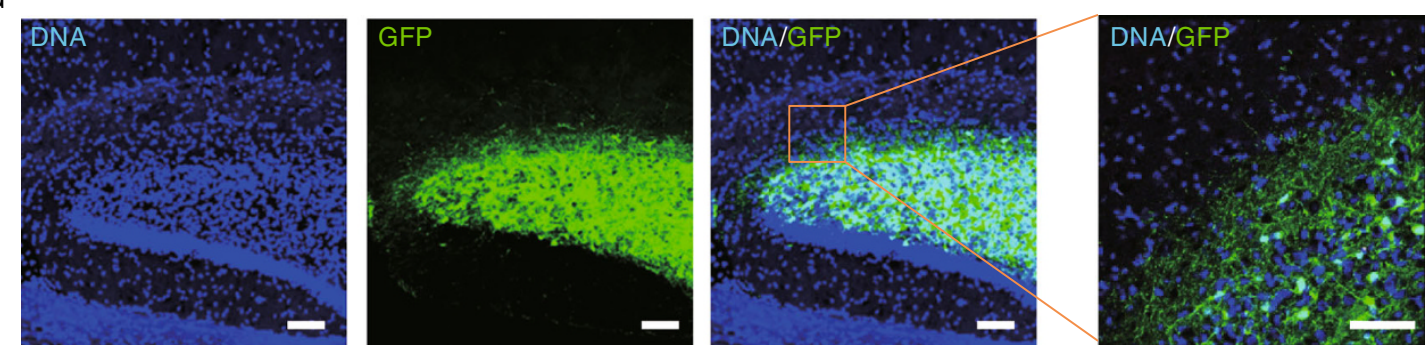
Figure 1. Direct reprogramming of porcine fetal fibroblasts (PFFs) to induced porcine neural progenitor cells (ipNPCs). (A) Immunofluorescence staining of ipNPCs derived on MEFs showing rosette morphology and expressions of neural progenitor cell markers Nestin (in green) and Sox2 (in red). Scale bars, $75 \mu \mathrm{m}$. (B) Immunofluorescence staining of Pax6 positive ipNPCs cultured on matrigel. Scale bars, $25 \mu \mathrm{m}$. (C) Gene expression analysis showed that the neural progenitor cell markers (NCAM, Nestin, Pax6) were induced in the direct conversion of PFFs to ipNPCs. All values are relative to PFFs and shown as mean \pm s.e.m. $(n=3)$. ${ }^{* *} P<0.001$. (D) Differentiation of ipNPCs into neurons (Tuj1, Green) and glial cells (GFAP, Yellow) in vitro. Scale bars, $100 \mu \mathrm{m}$. (E) Quantitative PCR analysis showed the induction of neuronal specific marker (Tuj1), glial specific marker (GFAP), and oligodendrocyte marker (MBP) after spontaneous differentiation of ipNPCs. All values are relative to PFFs and shown as mean \pm s.e.m. $(n=3)$. ${ }^{* *} P<0.01,{ }^{* *} P<0.001$. (F) Representative traces of electrophysiology recording of differentiated neurons at day 30. Inward fast inactivating sodium currents and outward currents were observed in differentiated neurons by whole cell current recording (left). Action potentials (APs) were elicited by step-current injections (middle). Spontaneous APs firing from ipNeuron were recorded (right). (G) In vivo transplantation of ipNPCs. Overview of transplanted ipNPCs (GFP + , Green) cells in the dentate gyrus (DG) of brain from NOD/SCID mice at 4 weeks after ipNPCs transplantation. Blue, DNA; Green, GFP.

Immunofluorescence staining showed that the majority of differentiated cells were Tuj1 positive neurons while GFAP positive glial cells were also present (Fig. 1D). Correspondingly, the mRNA levels of Tuj1 and GFAP significantly increased after ipNPCs spontaneous differentiation (Fig. 1E). In addition, up-regulation of myelin basic protein (MBP), which is enriched in oligodendrocytes, was also observed by qPCR (Fig. 1E), suggesting a possible existence of oligodendrocytes in the differentiated derivatives. Taken together, our data indicated multipotent neural differentiation potential of ipNPCs.

Subsequently, in order to assess the functional membrane properties of ipNPC-derived neurons (ipNeurons), electrophysiology recordings were performed after thirty days of spontaneous differentiation. Whole-cell patch-clamp recordings on ipNeurons revealed voltage-dependent currents including rapidly inactivating inward currents and persistent outward currents in response to depolarization voltage steps, which reflected open and closed states of sodium channels and potassium channels respectively (Fig. 1F, left). Current clamp recordings demonstrated that the ipNeurons could generate action potentials, an evident membrane characteristic of excitable cells (Fig. 1F, middle). Meanwhile, spontaneous action potentials were also observed in differentiated ipNeurons (Fig. 1F, right). Collectively, these data demonstrated that ipNPCs are capable of differentiating into excitable neurons in vitro.

Finally, we explored the neural differentiation potency of ipNPCs in vivo. The ipNPCs were labeled with GFP by lentiviral vectors, and then transplanted into the dentate gyrus (DG) of NOD/SCID mice. Four weeks after transplantation, brains of recipient mice were sectioned and analyzed. We found that most GFP-labeled cells were localized in the DG region, indicating a robust survival of ipNPCs in vivo (Fig. 1G). We further observed GFP positive neurons with complex branching morphology that were present at neighboring zones of the DG region (Fig. 1G), which suggested that ipNPCs were able to effectively integrate into the local neural network after transplantation. No teratoma formation was observed in any mouse brains examined, which further supports the safety of ipNPCs in vivo. (Data not shown)

In summary, we report here a new strategy to obtain integration-free functional porcine neural progenitor cells by direct reprogramming of porcine fetal fibroblasts in vitro. For the first time, porcine neural progenitor cells were directly generated from somatic cells, and functionally characterized both in vitro and in vivo. Considering the importance of pigs as a model species, a sufficient supply of functional porcine neural progenitor cells are of great interest in translational medicine studies of neuroscience. However, the difficulties to establish porcine pluripotent stem cells including embryonic stem cells and integration-free iPSCs limit the production of porcine NPCs through traditional cell differentiation approaches (Wu et al., 2009; Rasmussen et al., 2011; Liu et al., 2012c; Fan et al., 2013). Therefore, how to obtain porcine NPCs directly from the somatic cells is attracting a lot of attention in the field. Similar to many other direct reprogramming methods, our strategy bypassed obstacles in establishing porcine pluripotent stem cells. Moreover, our method provided a robust and efficient way of generating porcine NPCs with low risk of tumor formation. To our knowledge, this is the first attempt to direct reprogram somatic cells into neural progenitor cells using the porcine species. As a promising species of model animals, the ipNPCs generated in our study may provide an exciting tool to bridge the present gaps in neuroscience studies between rodents and humans.

\section{FOOTNOTES}

We are grateful to April Goebl for manuscript editing. GHL is supported by the Strategic Priority Research Program of the Chinese Academy of Sciences (XDA01020312), National Basic Research Program (2014CB964600), the National Natural Science Foundation of China (Grant Nos. 81271266, 31222039, and 81330008), Thousand Young Talents program of China, National Laboratory of Biomacromolecules (2012kf02, 2013kf05, 2013kf11), and State Key Laboratory of Drug Research (SIMM1302KF-17). JQ is supported by National Basic Research Program (2014CB910500) and the National Natural Science Foundation of China (81371342). XLX is supported by the National Natural Science Foundation of China (31201111). WQZ is supported by the National Natural Science Foundation of China (81300261). LNS is supported by the National Natural Science Foundation of China (81300677). DG is supported 
by China Postdoctoral Science Foundation (2013M530751). K.S is supported by a California Institute for Regenerative Medicine fellowship. LL is supported by the National Basic Research Program (973 Program) (Nos. 2009CB941000 and 2011CBA01002) and the National Natural Science Foundation of China (31271587). JCIB was supported by G. Harold and Leila Y. Mathers Charitable Foundation, The Leona M. and Harry B. Helmsley Charitable Trust, The Glenn Foundation and The Ellison Medical Foundation.

All the authors (Xiu-Ling Xu, Ji-Ping Yang, Li-Na Fu, Ruo-Tong Ren, Fei Yi, Keiichiro Suzuki, Kai Liu, Zhi-Chao Ding, Jing Qu, Wei-Qi Zhang, Ying Li, Ting-Ting Yuan, Guo-Hong Yuan, Li-Na Sui, Di Guan, Shun-Lei Duan, Hui-Ze Pan, Ping Wang, Xi-Ping Zhu, Nuria Montserrat, Ming Li, Rui-Jun Bai, Lin Liu, Juan Carlos Izpisua Belmonte, and Guang-Hui Liu) declare that they have no conflict of interest.

All institutional and national guidelines for the care and use of laboratory animals were followed.

Xiu-Ling $\mathrm{Xu}^{1}{ }^{1 凶}$, Ji-Ping Yang ${ }^{1}$, Li-Na Fu ${ }^{1}$, Ruo-Tong Ren ${ }^{1}$, Fei $\mathrm{Yi}^{2}$, Keiichiro Suzuki ${ }^{3}$, Kai Liu ${ }^{4}$, Zhi-Chao Ding ${ }^{1}$, Jing Qu ${ }^{1}$, Wei-Qi Zhang ${ }^{1}$, Ying $\mathrm{Li}^{1}$, Ting-Ting Yuan ${ }^{1}$, Guo-Hong Yuan ${ }^{1}$, Li-Na Sui ${ }^{1}$, Di Guan ${ }^{1}$, Shun-Lei Duan ${ }^{1}$, Hui-Ze Pan ${ }^{1}$, Ping Wang ${ }^{1}$, Xi-Ping Zhu ${ }^{1}$, Nuria Montserrat ${ }^{5}$, Ming Li ${ }^{1}$, Rui-Jun Bai ${ }^{1}$, Lin Liu ${ }^{4}$, Juan Carlos Izpisua Belmonte ${ }^{3}$, Guang-Hui Liu ${ }^{1,6 \bowtie}$

${ }^{1}$ National Laboratory of Biomacromolecules, Institute of Biophysics, Chinese Academy of Sciences, Beijing 100101, China

2 Department of Molecular and Cellular Physiology, Stanford University School of Medicine, Stanford, CA 94305, USA

${ }^{3}$ Gene Expression Laboratory, Salk Institute for Biological Studies, 10010 North Torrey Pines Road, La Jolla, CA 92037, USA

${ }^{4}$ State Key Laboratory of Medicinal Chemical Biology, College of Life Sciences, Nankai University, Tianjin 300071, China

${ }^{5}$ Center for Regenerative Medicine in Barcelona, Dr. Aiguader 88, 08003 Barcelona, Spain

${ }^{6}$ Beijing Institute for Brain Disorders, Beijing 100069, China

$\bowtie$ Correspondence: xiulingxu@hotmail.com (X.-L. Xu), ghliu@ibp.ac.cn (G.-H. Liu)

\section{OPEN ACCESS}

This article is distributed under the terms of the Creative Commons Attribution License which permits any use, distribution, and reproduction in any medium, provided the original author(s) and the source are credited.

\section{REFERENCES}

Ezashi T, Telugu BP, Alexenko AP, Sachdev S, Sinha S, Roberts RM (2009) Derivation of induced pluripotent stem cells from pig somatic cells. Proc Natl Acad Sci USA 106:10993-10998
Fan N, Chen J, Shang Z, Dou H, Ji G, Zou Q, Wu L, He L, Wang F, Liu K et al (2013) Piglets cloned from induced pluripotent stem cells. Cell Res 23:162-166

Giorgetti A, Marchetto MC, Li M, Yu D, Fazzina R, Mu Y, Adamo A, Paramonov I, Cardoso JC, Monasterio MB et al (2012) Cord blood-derived neuronal cells by ectopic expression of Sox2 and c-Myc. Proc Natl Acad Sci USA 109:12556-12561

Li M, Suzuki K, Qu J, Saini P, Dubova I, Yi F, Lee J, Sancho-Martinez I, Liu GH, Izpisua Belmonte JC (2011) Efficient correction of hemoglobinopathy-causing mutations by homologous recombination in integration-free patient iPSCs. Cell Res 21:1740-1744

Lind NM, Moustgaard A, Jelsing J, Vajta G, Cumming P, Hansen AK (2007) The use of pigs in neuroscience: modeling brain disorders. Neurosci Biobehav Rev 31:728-751

Liu GH, Barkho BZ, Ruiz S, Diep D, Qu J, Yang SL, Panopoulos AD, Suzuki K, Kurian L, Walsh C et al (2011) Recapitulation of premature ageing with iPSCs from Hutchinson-Gilford progeria syndrome. Nature 472:221-225

Liu GH, Qu J, Suzuki K, Nivet E, Li M, Montserrat N, Yi F, Xu X, Ruiz $S$, Zhang $W$ et al (2012a) Progressive degeneration of human neural stem cells caused by pathogenic LRRK2. Nature 491:603607

Liu GH, Yi F, Suzuki K, Qu J, Izpisua Belmonte JC (2012b) Induced neural stem cells: a new tool for studying neural development and neurological disorders. Cell Res 22:1087-1091

Liu K, Ji G, Mao J, Liu M, Wang L, Chen C, Liu L (2012c) Generation of porcine-induced pluripotent stem cells by using OCT4 and KLF4 porcine factors. Cell Reprogram 14:505-513

Lujan E, Chanda S, Ahlenius H, Sudhof TC, Wernig M (2012) Direct conversion of mouse fibroblasts to self-renewing, tripotent neural precursor cells. Proc Natl Acad Sci USA 109:2527-2532

Rasmussen MA, Hall VJ, Carter TF, Hyttel P (2011) Directed differentiation of porcine epiblast-derived neural progenitor cells into neurons and glia. Stem Cell Res 7:124-136

Thier M, Worsdorfer P, Lakes YB, Gorris R, Herms S, Opitz T, Seiferling D, Quandel T, Hoffmann P, Nothen MM et al (2012) Direct conversion of fibroblasts into stably expandable neural stem cells. Cell Stem Cell 10:473-479

Vierbuchen T, Ostermeier A, Pang ZP, Kokubu Y, Sudhof TC, Wernig $M$ (2010) Direct conversion of fibroblasts to functional neurons by defined factors. Nature 463:1035-1041

Wu Z, Chen J, Ren J, Bao L, Liao J, Cui C, Rao L, Li H, Gu Y, Dai H et al (2009) Generation of pig induced pluripotent stem cells with a drug-inducible system. J Mol Cell Biol 1:46-54

Zhang K, Liu GH, Yi F, Montserrat N, Hishida T, Rodriguez Esteban C, Izpisua Belmonte JC (2013) Direct conversion of human fibroblasts into retinal pigment epithelium-like cells by defined factors. Protein Cell. doi:10.1007/S13238-013-0011-2

Electronic supplementary material The online version of this article (doi:10.1007/s13238-013-0015-y) contains supplementary material, which is available to authorized users. 